\title{
Cooperative awareness in VANETs: On ETSI EN 302 637-2 performance
}

\author{
Nikita Lyamin, Alexey Vinel, Magnus Jonsson and Boris Bellalta
}

\begin{abstract}
Cooperative awareness on the road is intended to support the road users by providing knowledge about the surroundings and relies on the information exchange enabled by vehicular communications. To achieve this goal the European Telecommunication Standard Institute (ETSI) delivered the standard EN 302 637-2 for Cooperative Awareness Messages (CAM). The CAM triggering conditions are based on the kinematics of the originating vehicle, which is checked periodically. In this paper, we show that the standardized ETSI protocol may suffer a decrease in communication performance under several realistic mobility patterns. The potential influence of the discovered phenomena on the IEEE 802.11p Medium Access Control (MAC) operation is studied.
\end{abstract}

Index Terms-VANET, Cooperative Awareness, Platooning, ETSI, ITS-G5.

\section{INTRODUCTION}

$\mathbf{I}$ ntelligent Transport Systems (ITS) are aiming to provide innovative services related to different modes of transport and traffic management, and enable various users to be better informed and make safer, more coordinated and smarter use of transport networks [1]. This is supposed to be achieved by integrating telecommunications, electronics and information technologies with transport engineering in order to plan, design, operate, maintain and manage transport systems. CooperativeITS (C-ITS) supports connectivity between road users. Road users in this context are all kind of road vehicles like cars, trucks, motorcycles, bicycles or even pedestrians, and roadside infrastructure equipment [2]. Thus, C-ITS is an important component of Intelligent Transportation Systems and aim at increased road safety, efficiency and driving comfort.

To enable inter-vehicle communications in the Dedicated Short Range Communications (DSRC) $5.9 \mathrm{GHz}$ band, IEEE 802.11 , which is currently integrated into the recent IEEE 802.11-2012 standard, has been introduced by the Institute of Electrical and Electronics Engineers (IEEE). IEEE 802.11p provides the medium access control (MAC) and physical (PHY) layers for wireless communications in a vehicular environment. The IEEE 1609 working group has defined the protocol stack IEEE 1609.x, also known as WAVE (Wireless Access in Vehicular Environment). The scope of these standards is the extension of the IEEE 802.11p MAC layer functions for multichannel operation as well as the specification of the upper layers, functionality in security and management planes.

Nikita Lyamin, Alexey Vinel and Magnus Jonsson are with School of Information Technology, Halmstad University, Sweden

Boris Bellalta is with Department of Information and Communication Technologies, Universitat Pompeu Fabra, Spain

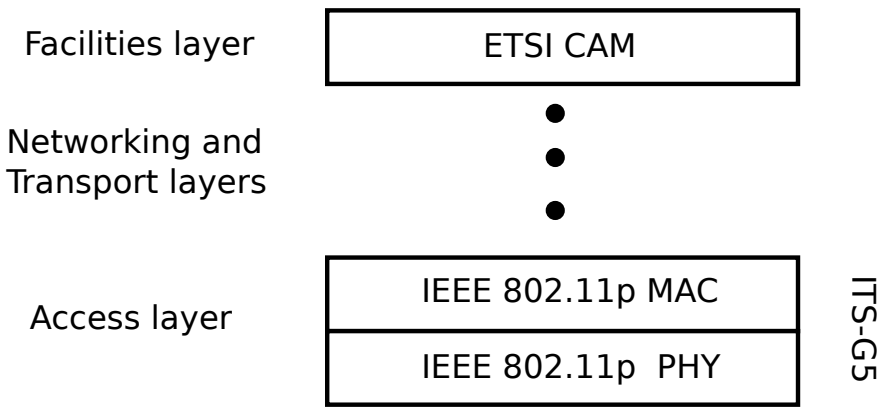

Fig. 1: ITS-G5 reference architecture

At the same time, European Telecommunication Standard Institute (ETSI) delivered the first release of the C-ITS standards under the European Commission Mandate M/453. ETSI specified the first set of ITS-G5 communication protocols and architecture regulating operation in the $5 \mathrm{GHz}$ spectrum for C-ITS, Fig. 1. ITS-G5 reuses the IEEE 802.11p physical and data-link layers of the IEEE 1609 framework. ITS-G5 also defines protocols to support cooperative awareness of road users, which is intended as the basis for a number of road safety and traffic efficiency applications [3]. It is achieved by regular exchange of information among road users. ETSI defines the cooperative awareness within road traffic in terms of when road users and roadside infrastructure are informed about each other's position, kinematics and attributes. To enable cooperative awareness within ITS-G5, ETSI delivered the EN 302 637-2 standard defining Cooperative Awareness Messages (CAMs) [2]. Note, that Cooperative Awareness basic service is mandatory for all kind of ITS-stations (ITS-S) operating in ITS-G5. Each ITS-S puts kinematic data and other related data into periodically sent CAMs. The content of the message may vary depending on the type of ITS-S. In this paper we focus on the cooperative awareness on the road and the vehicles as ITS-S.

EN 302 637-2 defines a kinematically-driven mechanism that controls CAM triggering. This means, that each vehicle generates new CAMs depending on its current position, speed and direction. A vehicle compares its current kinematic measurements with the ones it put into the last generated message and, if the difference between them is above predefined thresholds, the vehicle triggers next CAM transmission. The reason for this is to allow the vehicle to trigger more messages when its behavior is highly dynamic and vice versa. In other words, a vehicle transmits fewer messages when its behavior is predictable, and more messages when accelerating/decelerating, turning or driving at high speed. 
However, as a consequence, the ETSI CAM protocol has a behaviour that is much more difficult to analyze compared to traditional beaconing approaches that have a fixed frequency of message generation.

In this paper we study the communication performance of the EN 302 637-2 CAM mechanism under realistic mobility patterns for both autonomous and human-driven vehicles. Specifically, we assume two scenarios: Scenario 1. platoon of autonomous vehicles where coordination is supported by exchanging CAMs, and Scenario 2. string of human driven vehicles approaching a traffic light while exchanging CAMs to support cooperative awareness.

The contribution of this paper is threefold:

- The phenomenon of message synchronization in the time domain in adaptive beaconing relying on speed variation tracking of originated ITS-s is theoretically analyzed.

- The performance of the ETSI EN 302 637-2 standard is evaluated by considering two typical mobility patterns (autonomous vehicles and human-driven vehicles approaching a pedestrian crossing). The negative impact of the synchronization of CAM generation moments (initially found in [4], [5]) is further discussed, analyzed and evaluated.

- The influence of various ETSI EN 302 637-2 parameters in the synchronization of CAM generation moments is studied, and recommendations are given on how to mitigate the negative effect of CAM synchronization are given.

The manuscript is organized as follows. Section II overviews the related work. Section III summarizes the studied current ITS-G5 standardization activity. In Section IV, we describe the system model, including the principal assumptions about the inter-vehicle communication and vehicles' mobility. Section V explains the discovered phenomena of CAM synchronization. In Section VI, we propose a theoretical framework to evaluate the influence of the discovered CAM synchronization effect on communication performance. In Section VII, we demonstrate the influence of CAM synchronization on typical mobility patterns for both autonomous and humandriven vehicles under different ETSI EN 302 637-2 parameters setup. Conclusions are presented in Section VIII.

\section{RELATED WORK}

Here we overview the most relevant (in terms our study) state-of-the-art research into adaptive beaconing in C-ITS.

In [6] the authors provide an extensive survey of adaptive beaconing for C-ITS. They provide a taxonomy of adaptive beaconing approaches, summarize performance metrics used for their design, and present their qualitative comparison.

In [7] the authors propose an ATB (Adaptive Traffic Beacon) adaptive beaconing protocol for C-ITS that adapts its beacon generation rate based on two metrics - channel quality and message utility. To estimate channel quality, the authors propose the use of collision statistics collected by the ITS$\mathrm{S}$, the Signal to Noise Ratio (SNR) levels measurements, and the number of neighboring ITS-S. Message utility is expressed through message age and beacon target dissemination range.
Adaptive beaconing for enhancing the cooperative awareness by minimizing the tracking error of ITS-s is presented in [8]. The simulation results presented in the paper demonstrate that the proposed protocol outperforms ETSI CAM beaconing, supporting the lower figures of ITS-S tracking error. In [9], a Dynamic Beaconing Scheduling (DBS) adaptive beaconing approach based on the current kinematics of the ITS-S is presented. The beacon generation interval is proportional to the speed of the originating ITS-S: the higher the speed, the lower the beacon generation interval. The main idea of the proposed protocol is very similar to ETSI CAM kinematic rules, however, the authors do not provide any comparison with the performance of DBS and CAM. The adaptive beaconing protocol AND (Adaptive Neighbor Discovery) is presented in [10] and controls the beacon generation interval in order to maximize the discovery rate of the neighboring ITS-S in the specified road area. The simulation experiment performed by the authors shows that AND outperforms ETSI CAM in terms of ITS-S discovery accuracy at higher channel noise levels, while demonstrating similar performance when packet loss rates are low. In [11] the authors propose FABRIC (Fair Adaptive Beaconing Rate for Intervehicular Communications) - an algorithm that enables fair beaconing rate assignment for ITS-S. In FABRIC the transmission rate of each ITS-S in the one-hop neighborhood is recursively optimized. To enable this, it is assumed that all ITS-S share its beacon generation rate. The set of simulation experiments presented in the paper demonstrates that FABRIC has a fast convergence to fair beacon generation rate even in a highly dynamic environment.

ETSI CAM beaconing is an example of adaptive beaconing design for C-ITS to enable cooperative awareness in the vehicular environment. To adapt the CAM generation rate it adjusts the beaconing rate based on the current kinematics of the originating ITS-S.

Many studies on cooperative awareness either ignore ETSI kinematic rules [12]-[14] or implement CAM protocol according to the standard, but do not focus on its performance. The evaluation of CAM beaconing under various parameter sets is performed in [15]-[18]. In [19], the authors evaluate the CAM rules to understand the actual beaconing rate and corresponding channel load in a highway scenario. In [20], the authors present a more detailed study of the ETSI CAM kinematic rules. They attempt to find optimal parameters thresholds to enhance the network performance in terms of packet delivery ratio (PDR), channel load and message age.

Very few studies focusing on assessing the effectiveness of the rules proposed by ETSI for CAM generation. In [21] the applicability of ETSI EN 302 637-2 to support platooning was studied. The main conclusion was that CAM may support cooperative autonomous driving, while having gaps in application functionality: support of platooning merging/disaggregation, and, importantly, the lack of an appropriate authentication mechanism that can be used for secure platooning aggregation. The study concludes that improvements of the CAM data structure are necessary.

The authors of [22] present results of an extensive measurement study estimating the performance of CAM cooperative awareness in terms of neighborhood awareness ratio and 
packet delivery rate. The paper provides substantial results on CAM ability to support awareness at a certain level depending on various factors (environment, transmission power level, beaconing generation frequency, etc.). However, they discuss the results in terms of averaged performance metrics and do not focus on the functioning of the CAM generation mechanism's itself.

In [4], [5] the negative effect of CAM synchronization in a platooning scenario was identified. It was shown that in a string of vehicles under synchronous acceleration/deceleration maneuvers CAM generation times may synchronize and lead to an increase of the CAM collision rate. The current study examines the side effect under typical mobility patterns of autonomous and human-driven vehicles within various sets of CAM parameters. We evaluate the strength of such an effect under various conditions and give recommendations on how it may be avoided.

\section{STANDARDIZATION}

\section{A. ETSI EN 302 637-2}

The process of triggering CAMs is controlled by the Cooperative Awareness Basic Service [2] and can be described as follows ${ }^{1}$. The time between two consecutive generated CAMs is controlled within:

- $T_{\min }=T_{-}$GenCamMin $=100 \mathrm{~ms}$ (all the notions used throughout the paper are summarized in Table I), which is an upper bound corresponding to the maximum CAM generation rate of $10 \mathrm{~Hz}$. The time between two consecutive CAMs shall not be less than $T_{\min }$.

- $T_{\max }=T_{-}$GenCamMax $=1000 \mathrm{~ms}$, which is an upper bound corresponding to the minimum CAM generation rate of $1 \mathrm{~Hz}$.

Within these bounds CAMs shall be generated depending on the vehicle's kinematics. A vehicle repeatedly every $\Delta=T \_$CheckCamGen checks the deviation of its current speed, position and direction from the measurements that have been placed in the last triggered CAM. We refer to $1 / \Delta$ as the CAMs triggering sampling rate. A new CAM should be triggered if one of the following deviations has been observed:

- "Event A": the absolute difference between the current position of the vehicle and its position included in the previous CAM exceeds $d_{\min }=4 \mathrm{~m}$;

- "Event B": the absolute difference between the current speed and the speed included in the previous CAM exceeds $v_{\min }=0.5 \mathrm{~m} / \mathrm{s}$;

- "Event $C ":{ }^{2}$ the absolute difference between the current direction of the vehicle and the direction included in the previous CAM exceeds $4^{\circ}$.

\footnotetext{
${ }^{1}$ Subsequently, CAMs transmission could also be influenced by the ETSI Decentralized Congestion Control (DCC) [23]. However, throughout of this paper DCC is not considered. We exclude DCC to focus on the CAM synchronization effect only and make the analysis presented in the paper easier and more self-explanatory.

${ }^{2}$ Event $\mathrm{C}$ is not considered in the paper, since we assume that the vehicles move along the straight route or change its direction slowly. Nevertheless, all the presented considerations and conclusions are valid also in case Event $\mathrm{C}$ might occur.
}

The CAM shall be triggered if the time elapsed since the last CAM generation is greater than or equal to $T_{\max }$. Finally, we set $N_{\text {GenCam }}$, i.e. the number of subsequently triggered CAMs with a fixed current period after kinematic event is detected, to be equal to 1 .

\section{B. IEEE 802.11p MAC}

IEEE Std 802.11 offers several PHY layers and one common MAC sub-layer. At the same time, ETSI delivered the ITSG5 standard that specifies the two lowest layers to enable vehicle-to-vehicle communications in an ad-hoc network [24]. As mentioned above, the ITS-G5 standard is reusing the IEEE 802.11p MAC and PHY layers, Fig. 1.

In IEEE $802.11 \mathrm{p}$, ITS-S accesses to the media is controlled by the CSMA/CA (Carrier sense multiple access with collision avoidance) function. Before each transmission, a station picks up a random $\mathrm{BO}$ (backoff) value from the $[0, W]$ range. Provided the channel remains idle in the current time-slot, the $\mathrm{BO}$ value is decremented. The transmission starts when the $\mathrm{BO}$ value turns to zero. If the station identifies the channel as busy, it "freezes" the current BO value and starts to decrement it again after it detects the end of the ongoing transmission on the channel. Note that CAM is a broadcast message, which means that no acknowledgment or retransmissions are considered during its exchange.

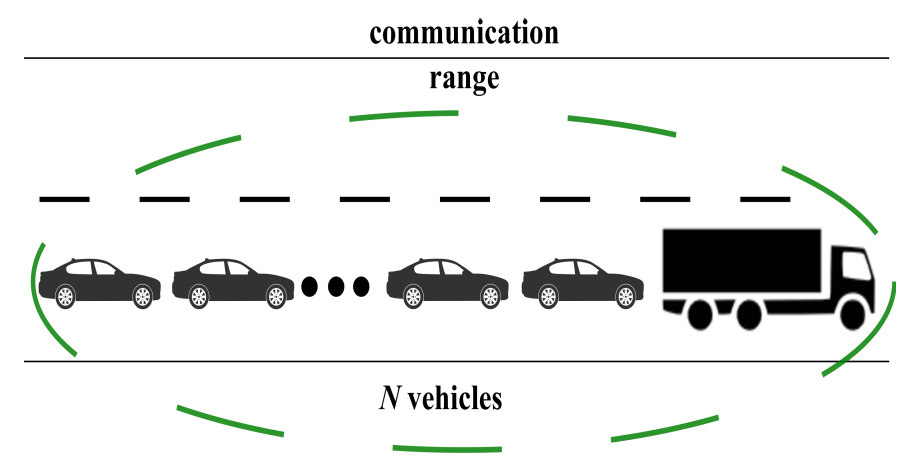

Fig. 2: Platooning (Scenario 1)

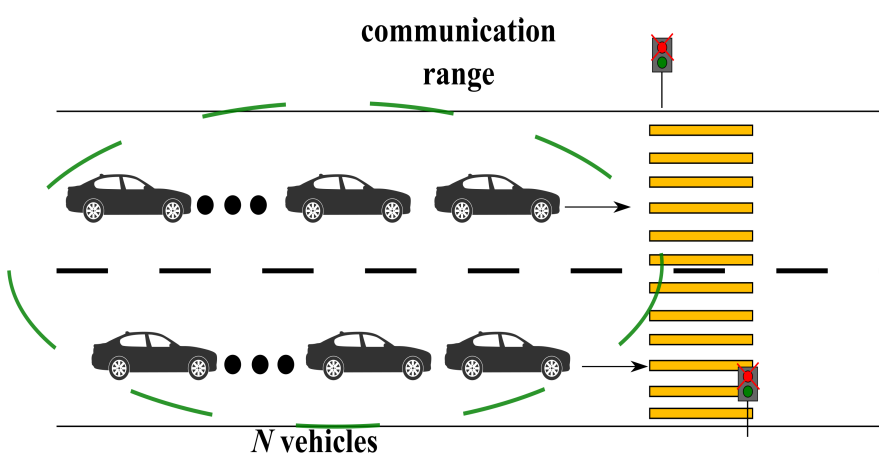

Fig. 3: Traffic jam (Scenario 2)

\section{SYSTEM MODEL}

In this study we focus on the following two mobility scenario, representing the cases of both autonomous and humandriven caravans of vehicles on the road. 
TABLE I: Main Notations

\begin{tabular}{|c|c|c|}
\hline Parameter & Value & Meaning \\
\hline$\Delta$ & - & CAM sampling period \\
\hline$d_{\min }$ & $4 \mathrm{~m}$ & "Event $A$ " threshold \\
\hline$v_{\min }$ & $0.5 \mathrm{~m} / \mathrm{s}$ & "Event B" threshold \\
\hline$W$ & 16 & contention window size \\
\hline$A I F S$ & $110 \mu \mathrm{s}$ & arbitration inter-frame spacing \\
\hline$T_{\min }$ & $100 \mathrm{~ms}$ & $\begin{array}{l}\text { minimum time allowed between two consec- } \\
\text { utive CAMs }\end{array}$ \\
\hline$T_{\max }$ & $1 \mathrm{~s}$ & $\begin{array}{l}\text { maximum time allowed between two consec- } \\
\text { utive CAMs }\end{array}$ \\
\hline$N$ & 25 & number of vehicles in the caravan \\
\hline$\sigma$ & $13 \mu \mathrm{s}$ & IEEE 802.11p aTimeSlot \\
\hline $\mathrm{V}_{s t b}$ & $25 \mathrm{~m} / \mathrm{s}$ & stable speed in the disturbance scenario \\
\hline $\mathrm{V}_{\text {low }}$ & - & low speed in the disturbance scenario \\
\hline$D$ & - & platoon desynchronization factor \\
\hline$v$ & - & current speed of the vehicle \\
\hline$t$ & - & current time value \\
\hline$t_{i}$ & - & $\begin{array}{l}\text { time when the most recent CAM was gener- } \\
\text { ated by the } i \text {-th vehicle }\end{array}$ \\
\hline$V_{i}$ & - & speed of the $i$-th vehicle at time $t_{i}$ \\
\hline$t_{\text {event }}$ & - & $\begin{array}{l}\text { actual time either "Event } A \text { " or "Event B" } \\
\text { occurred }\end{array}$ \\
\hline$\tau$ & - & $\begin{array}{l}\text { period with which the vehicle triggers CAMs } \\
\text { when moving at a constant speed }\end{array}$ \\
\hline$T_{C A M}$ & - & CAM transmission duration \\
\hline$Q(m)$ & - & $\begin{array}{l}\text { probability density function (PDF) of the } \\
\text { number of groups containing exactly } m \text { ve- } \\
\text { hicles }\end{array}$ \\
\hline$Q^{*}(m)$ & - & empirical $Q(m)$ \\
\hline$R$ & $3 \mathrm{Mbit} / \mathrm{s}$ & datarate \\
\hline$L$ & 400 bytes & length of CAM message \\
\hline
\end{tabular}

To support coordination and awareness between vehicles on the road, each vehicle executes the following steps:

- Generate CAMs in accordance with the ETSI EN 302 637-2 specification [2].

- Transmit CAMs on a dedicated channel in accordance with the IEEE 802.11p MAC specification [25].

\section{A. Scenario 1: platooning}

In this scenario we consider a platoon of CACC-enabled (Cooperative Adaptive Cruise Control) vehicles moving on a highway. In platooning/CACC the leading vehicle is driven by a human driver, while the following vehicles automatically maintain the velocity of the leading one, but their directions are still controlled by the drivers. Platooning aims to reduce the air-drag in the caravan of heavy-duty vehicles, which could significantly improve fuel consumption, while CACC is contributes mainly to the driving experience by enabling comfort through semi-autonomous driving [26]. Since, in terms of the focus of this study, there is no difference between the discovered phenomena both for CACC and platooning, we refer henceforth to this class of applications as platooning. The cooperation between the vehicles in the platoon is achieved by the frequent exchange of periodic broadcast messages, which we refer to as beacons. CAMs are European implementation of the beacons for ITS-G5. CAMs may contain various related information like the vehicle's ID and kinematic information on a vehicle, such as its current speed, position, direction, etc.

In the scenario shown in Fig. 2, the leading vehicle decelerates from the desired steady speed $\left(\mathrm{V}_{\text {stb }} \sim 90 \mathrm{~km} / \mathrm{h}\right)$ to a lower speed $\left(\mathrm{V}_{\text {low }} \sim 60 \mathrm{~km} / \mathrm{h}\right)$, maintains this speed for some time, and then accelerates back to the initial speed, see Fig. 4. This disturbance scenario could be regarded as a pattern to describe the appearance of a slow moving vehicle in front of the platoon (coming from another lane or a metering ramp) or a road speed limit.

We consider a platooning system consisting of $N$ vehicles, where the leading vehicle is driven by a human driver, while the remaining $N-1$ vehicles are following automatically. On the basis of our previous works [4], [5], [27], the following assumptions are made in the present study:

- All the vehicles in the platoon are within each other's communication range. This is a valid assumption since for the inter-vehicle distance of $7 \mathrm{~m}$, which for 20-25 vehicles results in a maximum platoon size as of $300 \mathrm{~m}$ when the car length is $5 \mathrm{~m}$, and less than $500 \mathrm{~m}$ when the truck size is $13 \mathrm{~m}$. A recent measurement exercise (see Fig. 4, [28]) shows that in a convoy of vehicles moving on a highway, exploiting IEEE 802.11p transceivers in the $5.9 \mathrm{GHz}$ band, the communication range, where ITS-S experiences confident packet reception, is at least $500 \mathrm{~m}$.

- We assume noise-free channel and exclude the complementary influence of the CAM losses caused by channel impairments, since they would have no impact on the CAM synchronization effect, which is our focus.

- The kinematic parameters of the leading vehicle are modeled via the intelligent driver model (IDM) state-ofthe-art car-following mobility model [29].

- Since in reality vehicles in the platoon are not perfectly synchronized in their maneuvers, i.e., to assess the influence of the CAM synchronization in a more realistic setup, we add disturbance in the coordination between vehicles in the platoon. Random deviations in the velocities of the following vehicles with respect to the leading one are modeled by applying the following approach: we add a uniformly distributed random delay $\sim$ uniform $[0, D \cdot \sigma]$ to a CAM generation moment in order to reflect the non-perfect synchronization between their velocities, where $D$ is the maximum delay expressed in $\sigma=$ aTimeSlot (aTimeSlot is defined in the standard [25]). We refer to $D$ as a desynchronization factor.

\section{B. Scenario 2: traffic jam}

In this scenario, we consider a set of vehicles moving on the road with no coordination between them, while still exchanging CAM messages, Fig. 3. According to [2] "The Cooperative Awareness (CA) basic service is a mandatory facility for all kind of ITS-Stations (ITS-S), which take part in the road traffic". In other words, all the vehicles equipped with DSRC must participate in the CAM exchange. By analogy with scenario 1 in this paper, we focus on the disturbance scenario described above. In case of scenario 2, the disturbance scenario emulates a string of vehicles approaching traffic 


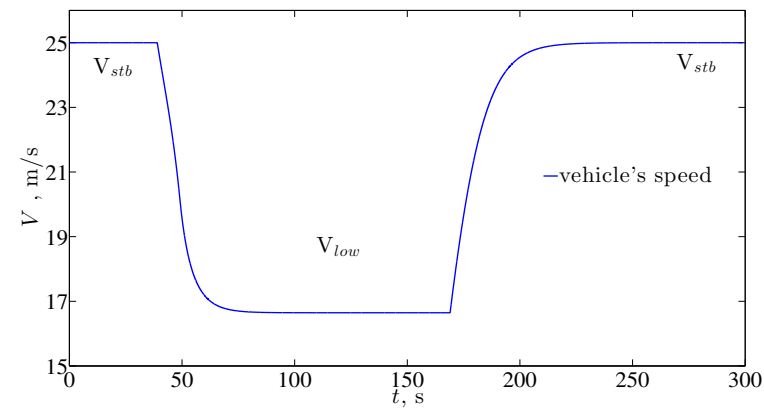

Fig. 4: Platoon speed variations due to a temporary obstacle as modeled via the $\mathrm{CAH}$ model with recommended comfort parameters [29] (Example 1)

lights. In the situation where the lights switch to red, the vehicles closest in the intersection at the road lanes start to decelerate roughly at the same time. Vehicles decelerate until they completely stop at the intersection, wait until the lights turn green and accelerate again to cross the intersection. Thus, in this scenario vehicles again follow the disturbance scenario shown in Fig. 2, with $\mathrm{V}_{\text {low }}=0 \mathrm{~km} / \mathrm{h}$. Note that, since the vehicles have human drivers without any additional coordination between them, each vehicle behind starts to decelerate with some delay caused by the driver's reaction time, inter-vehicle gap, etc.

In this scenario we consider several vehicles moving on a multi-lane road. All the vehicles are equipped with transceivers and periodically broadcast CAMs. The assumptions in scenario 2 are as follows:

- All the vehicles are within the same communication range, e.g., we consider $500 \mathrm{~m}$ distance prior to the traffic lights, assuming the IEEE $802.11 \mathrm{p}$ communication range is in the order of $400-500 \mathrm{~m}$.

- First vehicles on the road lanes (those that are closest to the traffic lights) react to the stoplight simultaneously, while the rest of the vehicles in the lane following them have no C-ITS-enabled coordination.

- The kinematic parameters of all the vehicle are modeled independently via the IDM mobility model.

\section{IDENTIFIED PHENOMENA: SYNCHRONIZED GENERATION OF CAMS}

\section{A. Discovery of the phenomenon in system-level simulations}

To illustrate the appearance of the CAM synchronization phenomenon and its negative impact we first set up a platooning experiment in the Plexe/Veins simulation environment [30]. Plexe is the detailed state-of-the art system level platooning simulator which incorporates tightly-coupled mobility, automatic control and communication components. We simulate a platoon of $N=15$ vehicles enabled by CAM exchange enabled by ETSI EN 302 637-2. The platoon moves along the straight stretch of a highway with a target speed for the leading vehicle $V_{s t b}=27.7 \mathrm{~m} / \mathrm{s}(\sim 100 \mathrm{~km} / \mathrm{h})$. Each vehicle in the platoon adapts its speed based on the kinematic information received from the platoon leader and the preceding vehicle. We use a longitudinal control algorithm based on the sliding surface method of the controller design presented in [31]. Two additional slower vehicles are inserted in front of the platoon at simulation time $200 \mathrm{~s}$ and $286 \mathrm{~s}$, Fig. 5. Thus, the platoon approaches slower vehicles and performs appropriate acceleration or deceleration maneuvers according to the disturbance scenario.

Results presented in Fig. 5 provide us with an evidence that the CAM collisions before maneuver are very unlikely to appear. However, after the platoon decelerates and accelerates the number of collisions observed grows significantly. From this we can conclude that certain mobility patterns may lead to a degradation in communication performance of the platoon enabled by CAM. Our hypothesis is that CAM messages of different vehicles become synchronized due to the operation of ETSI CAM kinematic triggering rules.

\section{B. Explanation of the CAM synchronization phenomenon}

To illustrate the phenomenon of CAM synchronization we study the stream of perfectly synchronized vehicles (all the vehicles in the stream perform acceleration/deceleration maneuvers simultaneously). This pattern could be considered as a perfect operation of a stream of CACC/platooning vehicles. Throughout this section we refer to this mobility pattern as platoon. We also set the value of the CAM sampling period to a very small value $\Delta=\sigma$ to allow vehicles to track precisely the occurrence of CAM kinematic events.

In the scenario shown in Fig. 4, the leading vehicle decelerates from the desired steady speed $\left(\mathrm{V}_{\mathrm{stb}} \sim 90 \mathrm{~km} / \mathrm{h}\right)$ to a lower speed $\left(\mathrm{V}_{\text {low }} \sim 60 \mathrm{~km} / \mathrm{h}\right)$, maintains this speed for some time, and then accelerates back to the initial speed.

Proposition $\boldsymbol{A}$. Let the platoon move with a constant speed $v$ and each vehicle triggers CAMs periodically (with period $\tau=d_{\text {min }} / v$ ) due to the occurrence of Event A. Consider a moment of time $t$ when the kinematic parameters of the platoon change so that Event B occurs for some vehicles. Let $\left\{t_{1}, t_{2}, \ldots t_{N}\right\}$ denote the moments of time when the most recent CAMs were generated by each vehicle in the platoon by time $t$. Then all the vehicles, for which the condition

$$
t-t_{i} \geq T_{\min }
$$

holds, will generate a new CAM at time $t$.

Proof: Because $t-t_{i}$ is the time elapsed since the most recent CAM generation by the $i$-th vehicle, the proposition directly follows from the CAM triggering rules.

To illustrate the effect of possible CAM generation times synchronization, let us consider two examples.

Example 1: Let the platoon change its velocity, e.g., it temporarily slows down due to a reduced speed limit in a road construction segment or due to a slow vehicle ahead, Fig. 4.

Let us denote the CAM generation moments of the $i$-th vehicle as $t_{1}, t_{2}, \ldots t_{N}$ and corresponding speeds as $V_{1}, V_{2}, \ldots V_{N}$, Fig. 6. When the platoon moves at a constant speed of $90 \mathrm{~km} / \mathrm{h}$, each vehicle triggers a CAM every $d_{\min } / V_{i}=160 \mathrm{~ms}$ due to the periodic occurrence of Event $A$.

Due to the deceleration, in a short time period the change in the platoon speed exceeds $0.5 \mathrm{~m} / \mathrm{s}$ (Event B) and the vehicles 

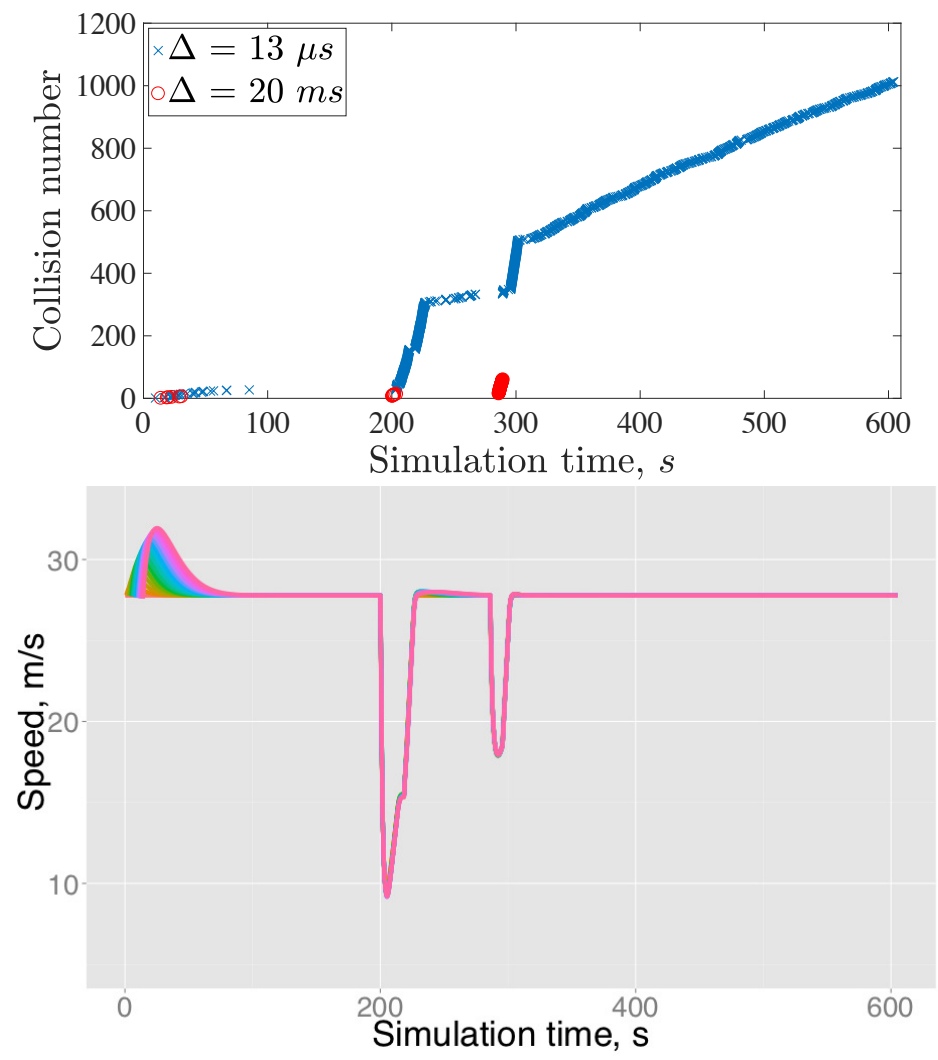

Fig. 5: Dynamics of CAM collisions during the maneuvering of a platoon modeled in Plexe

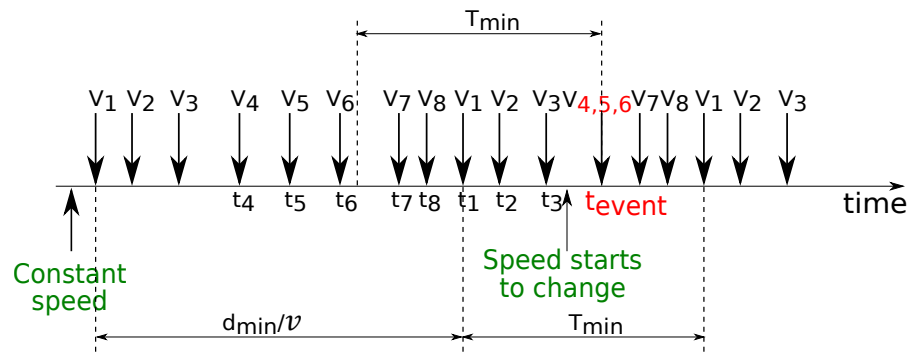

Fig. 6: Synchronization of CAM triggering moments in the CACC/platoon due to the synchronous speed changes (Example 1)

with $t_{\text {event }}-t_{i} \geq T_{\min }$ (i.e., 4, 5 and 6) synchronously trigger their CAMs at time $t_{\text {event }}$. Other vehicles (i.e., 1, 2, 3, 7 and 8) trigger their CAMs as soon as the time elapsed since their most recent CAM generation turns to $T_{\min }=100 \mathrm{~ms}$.

When the platoon speed stabilizes, the vehicles trigger CAMs with a constant period again (due to periodic occurrence of Event A).

Example 2: Let the platoon slow down and accelerate several times, see Fig. 8.

Each platoon maneuver will influence the CAM triggering process according to the mechanism described in Example 1. More CAMs might become synchronized as long as more maneuvers are performed due to the concurrent occurrence of Event B. For example, in Fig. 7 CAMs from vehicles 7, 8, 9 and 10 and 11,12 become synchronized with those from $1,2,3,4,5$ and 6 after the $2^{\text {nd }}$ and the $3^{r d}$ maneuvers, respectively.

Notice, that when vehicles in a caravan are perfectly synchronized and $\Delta$ is small once the synchronization of CAMs triggering times has occurred, further accelerations/decelerations will not lead to desynchronization. Event $B$ occurs simultaneously for all the synchronized vehicles, since their recent CAMs contain the same kinematic information.

\section{THEORETICAL ANALYSIS}

In Section V we explained the mechanism of CAM synchronization. Now we quantitatively characterize the discovered negative effect using:

- the mean number of the synchronized CAMs;

- the empirical PDF of the number of vehicles with the synchronized CAMs.

The results presented in this Section might be applicable to any adaptive beaconing approach where the speed variations of the originating ITS-S are used to trigger CAMs. 


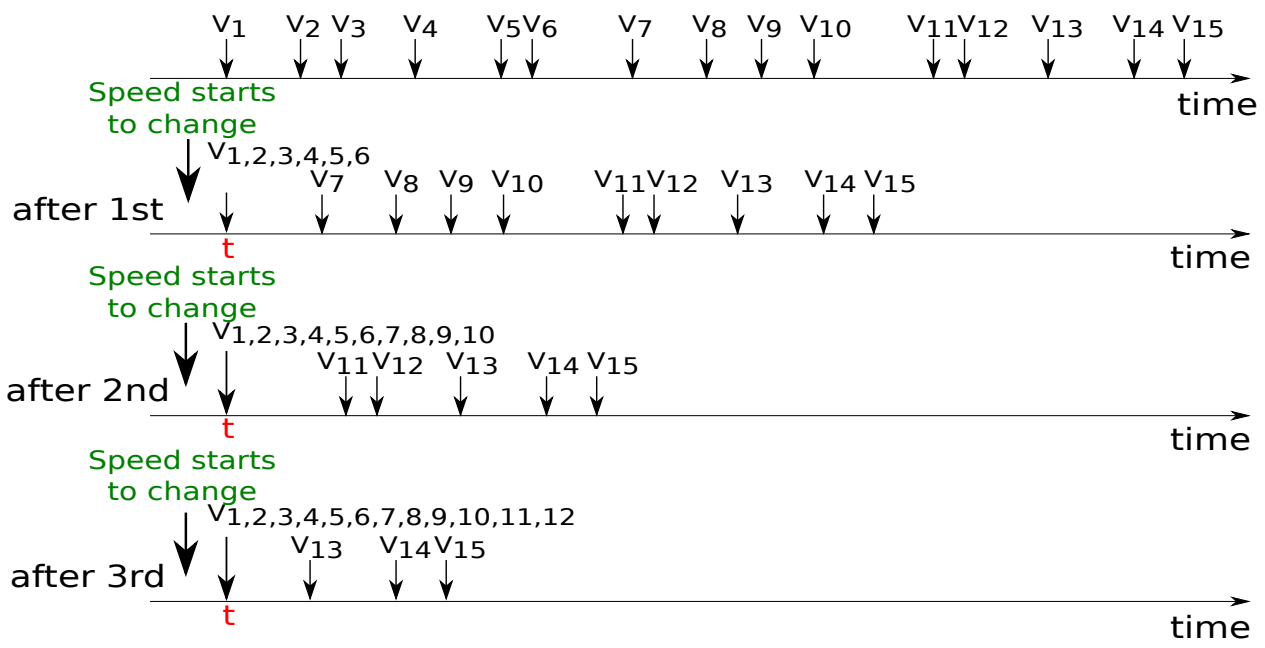

Fig. 7: Increased synchronization of CAM triggering moments after several maneuvers (Example 2)

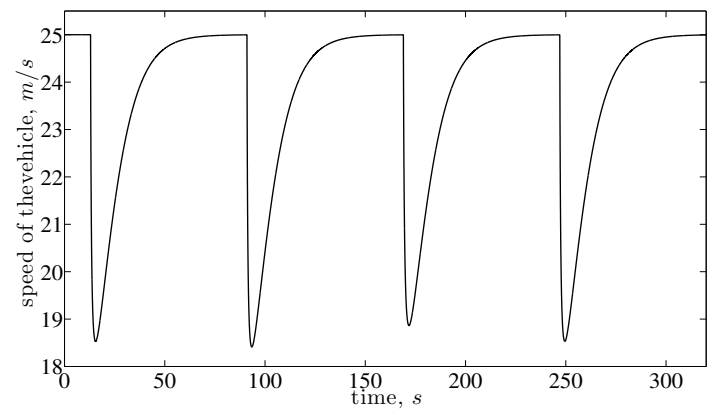

Fig. 8: Platoon speed variations in few subsequent deceleration/acceleration maneuvers (Example 2)

\section{A. Mean number of synchronized CAMs}

Let us present a simple analytical model, that estimates the mean number of synchronized CAMs in a string of perfectly synchronous vehicles driving according to the disturbance mobility pattern. Note, that this assumption is adopted to derive the closed-form expression for the mean number of synchronized CAMs. To model the disturbance in a platoon coordination we have introduced a desynchronization factor $D$, which is used to relax this assumption in Section VII).

Proposition B. Let a platoon of size $N$ move with at a constant speed $v$ during time interval $\left[0, t_{\text {event }}\right)$. If Event $B$ occurs at $t_{\text {event }}$ (instantaneous speed variation exceeds $v_{\text {min }}$ ), then the mean number of CAM generation moments synchronized at $t_{\text {event }}$ is

$$
\rho=\frac{\tau-T_{\min }}{\tau} \times N
$$

where $\tau=d_{\min } / v$.

Proof: Let the CAM generation moments of all the vehicles be enumerated and denoted as $t_{n}, n \geq 1$. The CAM generation moments in the interval $\left[0, t_{\text {event }}\right)$ represent the following stochastic process:

- Due to the random and independent occurrence of the first CAM generation moment of each of the $N$ vehicles, the $N-1$ intervals between pairs of subsequent CAMs of any $N$ consecutive generation moments are exponentially distributed, i.e. $\forall n: t_{n+k}-t_{n+k-1} \sim \exp (\tau / N), k=$ $\overline{1, N-1}$.

- Due to the periodic occurrence of Event $A$, all the vehicles generate CAMs with period $\tau$, i.e. $\forall n: t_{n+N+1}-t_{n}=$ $\tau$. Therefore, any time interval of duration $\tau$, contains exactly $N$ CAM generation moments (one per vehicle).

Due to the assumptions adopted in this Section, all $N$ vehicles detect Event $B$ simultaneously at $t_{\text {event }}$. However, due to the restriction on the value of the minimal possible CAM generation interval $T_{\min }$, only those vehicles, whose CAM generation moments belong to $\left[t_{\text {event }}-\tau, t_{\text {event }}-T_{\min }\right)$, are triggered at $t_{\text {event }}$, Fig. 9. Taking into account the above properties of the considered stochastic process, the mean number of CAM generation moments in $\left[t_{\text {event }}-\tau, t_{\text {event }}-T_{\min }\right)$ is $\frac{\tau-T_{\min }}{\tau} \times N$.

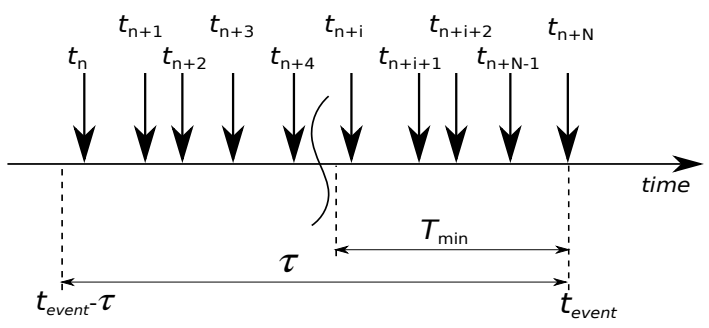

Fig. 9: The fraction of the CAM triggering moments (leftside) become synchronized after $t_{\text {event }}$

\section{B. Notion of groups}

Transmission of CAMs generated as discussed above is governed by the IEEE 802.11p MAC protocol, which presumes that CAMs from different vehicles may collide due to their simultaneous generation. Synchronization of the CAM generation times does not make a collision inevitable in the same way that their desynchronized generations do not make collisions impossible [32], [25]. This phenomenon can be characterized using the notions of groups.

Let us consider a platoon moving at a constant speed with all the vehicles periodically triggering CAMs. Let us select a sequence of $t_{i} \leq t_{i+1} \leq t_{i+2} \leq \cdots \leq t_{i+N-1}$ CAM 
generation moments of each vehicle in the platoon such that CAMs from vehicles $i$ and $i+N-1$ cannot collide (formal proof is proposed in [27], p. 112).

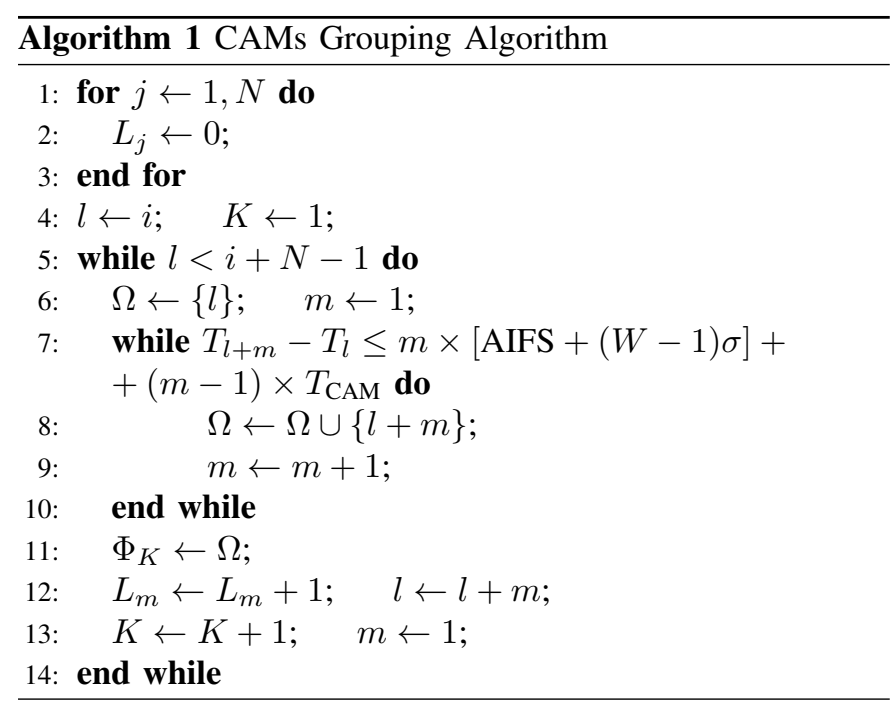

One can execute Algorithm 1, where AIFS is the Arbitrary Inter-Frame Space, $\sigma$ is a aSlotTime, $W$ is the Contention Window [25] and $T_{C A M}$ is the CAM transmission time. The outcome of the Algorithm operation is that all $N$ vehicles are split into $K$ sets denoted as $\Phi_{k}, k=1 \ldots K$ and further referred to as groups. $L_{m}$ is the number of groups consisting of exactly $m$ vehicles.

Proposition $\boldsymbol{C}$. The CAMs of vehicles belonging to different groups $\Phi_{k}(k=1 \ldots K)$ cannot collide.

Proof: From the IEEE 802.11p backoff rules it follows that in the empty system two CAMs can never collide if their generation moments are spread in time for at least AIFS $+(W-1) \sigma$, see line 7. When the CAMs are generated during the ongoing transmissions of other vehicles, the backoff counters freeze until the channel becomes idle. Respective maximum possible transmission delays are checked at line 7.

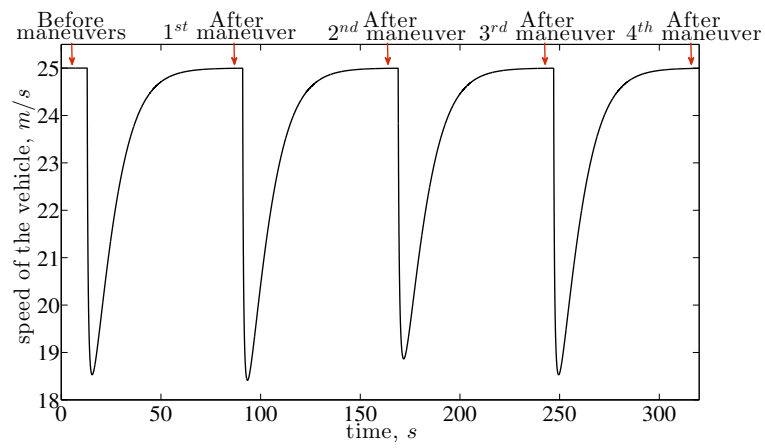

Fig. 10: Reference moments (indicated by the arrows) at which the CAM performance is assessed

The PDF of the number of groups with $m$ vehicles is defined as $Q(m)=\operatorname{Pr}\{x=m\}=L_{m} / K$.

For analysis purpose, let us consider time intervals where the speed of the vehicle is constant, i.e., before any maneuvers and after each of the maneuvers (see Fig. 10).
Since the results presented in the paper were obtained using simulations, we will operate with Empirical PDF $Q^{*}(m)$ for the number of groups with $m$ vehicles ${ }^{3}$. The results are obtained via simulations with standard IEEE 802.11 p parameters as in [27].

\section{EVAlUATION OF THE PHENOMENA}

In this Section we present the outcomes of our simulation study. First, we investigate a platoon enabled by inter-vehicular CAM exchange. We then discuss the influence of the CAM synchronization effect on a string of human driven vehicles supporting cooperative awareness on the road. The parameters of the study are summarized in Table II.

TABLE II: Simulation Parameters

\begin{tabular}{cc}
\hline \multicolumn{1}{c}{ Parameter } & Value \\
\hline$N$ & Scenario 1 \\
Number of road lanes & 25 \\
$V_{\text {stb }}$ & $25 \mathrm{~m} / \mathrm{s}(90 \mathrm{~km} / \mathrm{h})$ \\
$V_{\text {low }}$ & $18-19 \mathrm{~m} / \mathrm{s}(65-68 \mathrm{~km} / \mathrm{h})$ \\
$\Delta$ & $1 \cdot \sigma-1500 \cdot \sigma$ \\
$D$ & 500 \\
\hline & Scenario 2 \\
Number of road lanes & $25 \mathrm{per} \mathrm{lane}$ \\
$V_{\text {stb }}$ & 3 \\
$V_{\text {low }}$ & $16.7 \mathrm{~m} / \mathrm{s}(60 \mathrm{~km} / \mathrm{h})$ \\
$\Delta$ & $0 \mathrm{~m} / \mathrm{s}$ \\
& $1 \cdot \sigma$ \\
\hline
\end{tabular}

\section{A. Scenario 1: platooning}

In this scenario we independently study the influence of CAM sampling period $(\Delta)$ on the described synchronization effect first, and we assess the joint influence of $\Delta$ and the desynchronization factor $(D)$ between vehicles in the platoon.

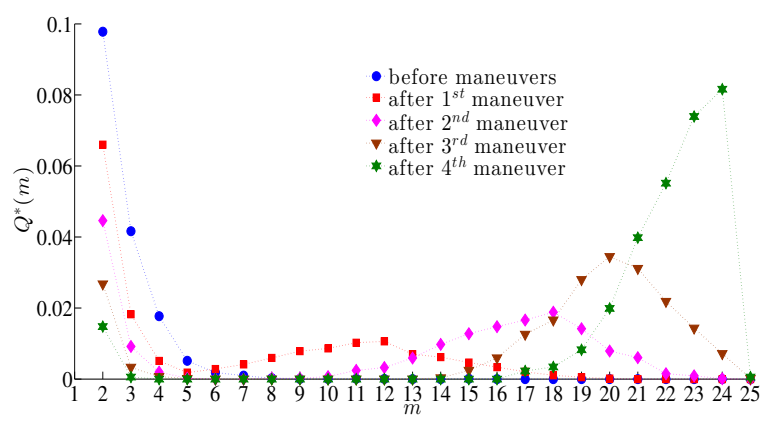

Fig. 11: Influence of maneuvers on CAM generation moments when $\Delta=1 \cdot \sigma$

In the first set of simulations reported in Fig. 11, the value of $\Delta$ is set to be $\Delta=\sigma$. When the platoon demonstrates a synchronous behavior and the sampling rate is high, after each subsequent maneuver the time diversity between CAMs

\footnotetext{
${ }^{3}$ For the sake of the plots clarity, the values of $Q^{*}(1)$ are not depicted.
} 
belonging to different vehicles continuously decreases. Thus, after the $4^{\text {th }}$ maneuver the CAMs belonging to almost all $N$ platoon members are in the same group. Although the formation of a group does not necessarily result in a collision of CAMs, the number of groups and the vehicles in each group could be directly related to the actual CAM collision probability. Fig. 18 shows the result of CAM collision probability for several setups. After each maneuver with the decrease of time diversity, collision probability grows accordingly.

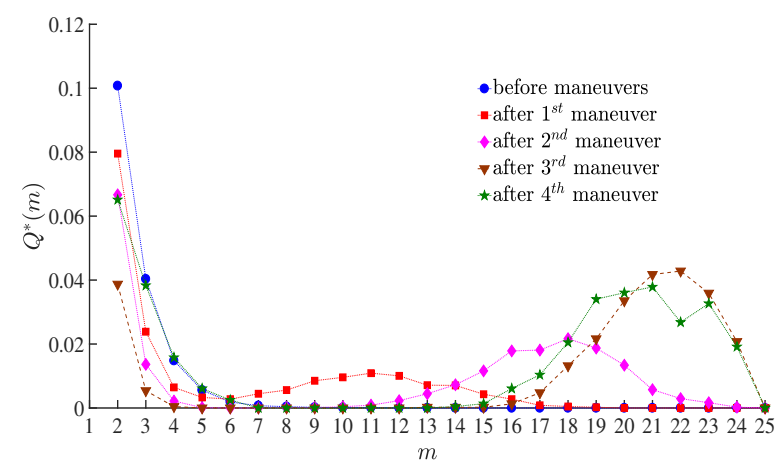

Fig. 12: Influence of maneuvers on CAM generation moments when $\Delta=100 \cdot \sigma$

Figs. $12-15$ demonstrate results when $\Delta$ is gradually increased. Increasing $\Delta$ decreases the strength of the effect accordingly. A larger value of $\Delta$ makes the reaction of platoon members to diverge in time. With the increase of $\Delta$, a vehicle detects the occurrence of the events with uniformly distributed delays uniform $[0, \Delta \sigma] \mathrm{ms}$. Thus, platoon members register the event at different points in time (depending on their own time they perform sampling). As a result the time each vehicle triggers CAM transmission lies within $\left[t_{\text {event }}, t_{\text {event }}+\Delta \sigma\right]$, where $t_{\text {event }}$ is the actual time when the message triggering event occurs. The larger $\Delta$, the larger the time interval during which all platoon members trigger their corresponding CAM. Example: when $\Delta=1500 \sigma$ (which corresponds to $\sim 20$ $\mathrm{ms}$ ), each vehicle performs samplings approximately every $20 \mathrm{~ms}$. This results in CAM transmission from each platoon member being triggered within $20 \mathrm{~ms}$ with some shift from actual $t_{\text {event }}$, which is caused by the random shift between the times each specific vehicle performs its samplings. At the same time, obviously, the increase of $\Delta$ results in increasing delays between a kinematic event and its registration.

Figs. 16-17 show the joint influence of sampling period $\Delta$ and the desynchronization factor of platoon members coordination on the discovered CAM synchronization effect. Our simulations show that possible disturbances in the coordination between platoon members can have a positive consequence, as it may significantly mitigate the CAMs synchronization phenomenon. From Figs. 16 and 18 one can conclude that $\delta$ may help to mitigate the CAMs synchronization effect significantly. Random disturbance acts in the same way as the random backoff mechanism in the CSMA/CA protocol, by actually, separating concurrent CAM generation in time, Fig. 18. In the case where CAMs were also diversified by larger $\Delta$, an additional random desynchronization component

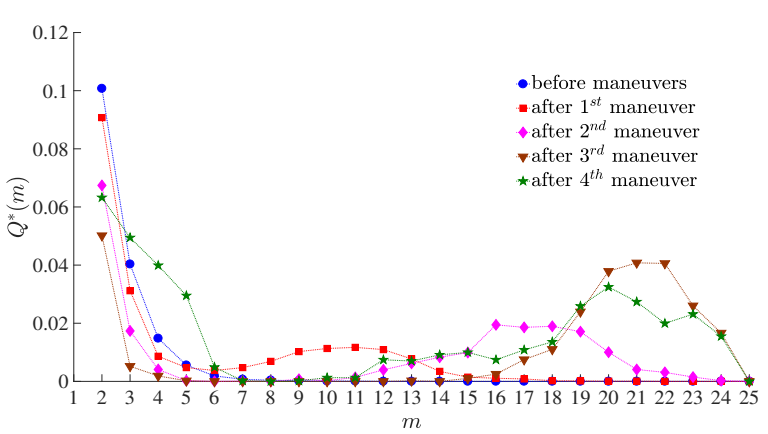

Fig. 13: Influence of maneuvers on CAM generation moments when $\Delta=200 \cdot \sigma$

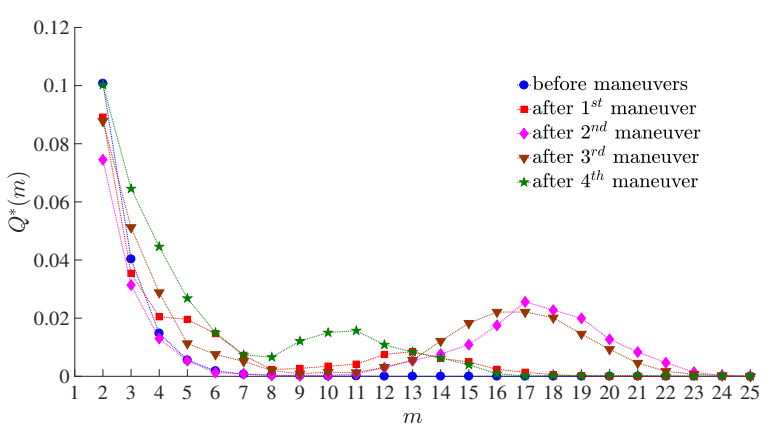

Fig. 14: Influence of maneuvers on CAM generation moments when $\Delta=500 \cdot \sigma$

can eliminate the CAM synchronization effect more or less completely, see figure 17.

Fig. 18 shows CAM collision probabilities for corresponding setups, presented on Figs. 11, 14, 16 and 17. It can be seen that the effect of synchronization of CAMs in time can be proportionally related to the actual CAM collision probability.

\section{B. Scenario 2: traffic jam}

In this scenario we consider independently driven vehicles approaching the traffic lights. We consider a 3-lane road with $N=25$ vehicles in each lane (75 vehicles in total), following a disturbance speed scenario with $\mathrm{V}_{\text {stb }}=60 \mathrm{~km} / \mathrm{h}$ and $\mathrm{V}_{\text {low }}=0$ $\mathrm{km} / \mathrm{h}$. Vehicles broadcast CAMs according to [2]. To study the synchronization of the CAMs generation moments we plot a histogram, where we place CAM triggering times belonging to the vehicles in time bins of $5 \mathrm{~ms}$ each for the duration of $0.5 \mathrm{~s}$. Following our approach, we show CAM synchronization after each subsequent maneuver, Figs. 19, 20, 21. As a benchmark for comparison, together with a plot for the corresponding maneuver, we also show the figure of CAM generation times before maneuvers. The simulations show that time diversity between CAM generation moments decreases after each subsequent maneuver, following the mechanism described in this paper. It is worth noting that in this scenario the strength of the CAM synchronization effect is noticeably lower due to the much higher independence in the maneuvers of the vehicles. However, the grouping of the CAM generation moments is still clearly visible. 


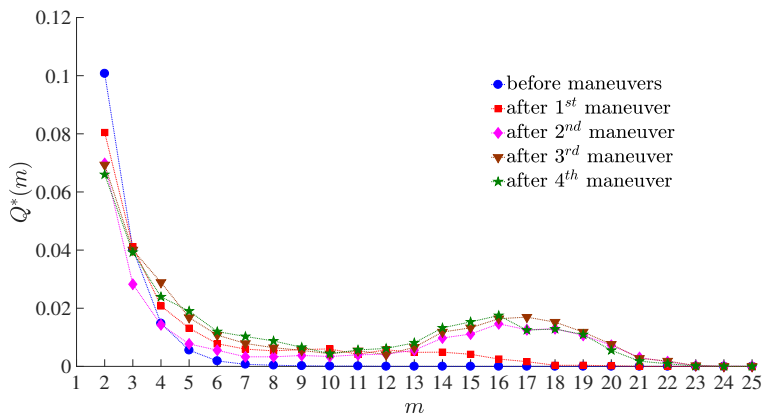

Fig. 15: Influence of maneuvers on CAM generation moments when $\Delta=1500 \cdot \sigma$

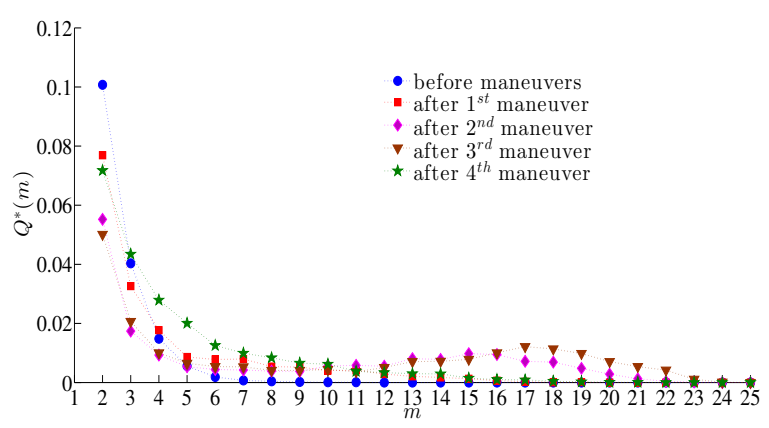

Fig. 16: Influence of maneuvers on CAM generation moments when $\Delta=1 \cdot \sigma, D=500$

We note, that if the traffic lights are equipped with a Road Side Unit (RSU) that broadcasts the "stoplight" to the vehicles approaching it, this will cause a simultaneous harmonized deceleration maneuver. In this case, the analysis presented for scenario 1 (CACC/platooning) CAM synchronization can be directly inherited by scenario 2 with corresponding conclusions regarding communication performance.

\section{CONCLUSIONS}

Enabling cooperative awareness requires extensive periodic exchange of kinematic information between vehicles. In its turn, this results in an extensive beaconing exchange. Our study has shown that adaptive beaconing based on the kinematc-driven CAMs has a potential performance drawback when implemented in cooperative applications that assume the coordinated behavior of the vehicles. The negative effect of CAM synchronization could be avoided by either:

- Decreasing the sampling rate (increasing $\Delta$ ). From our simulation study (Figs. 11-15) we conclude that for a value of the sampling period in the order of $\Delta=1500 \cdot \sigma$ (which is about $20 \mathrm{~ms}$ ) the CAM synchronization effect observed is considerably reduced.

- Decreasing synchronicity between vehicles (increasing the desynchronization factor). Random disturbance acts in the same way as the random backoff mechanism in the CSMA/CA protocol, by actually, separating concurrent CAM generation in time. In the case when CAMs are also diversified by larger $\Delta$, an additional random

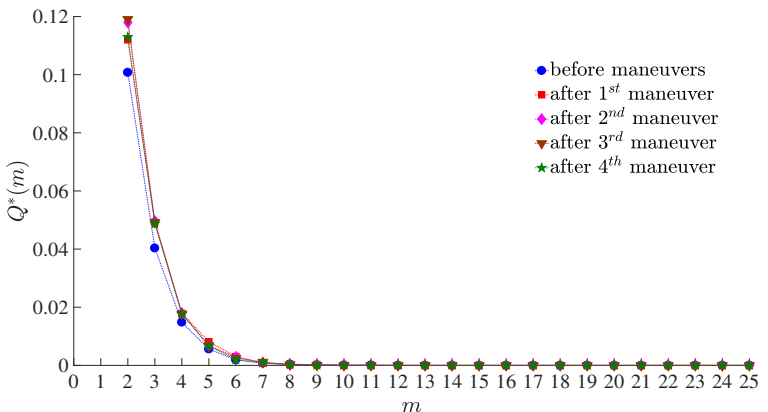

Fig. 17: Influence of maneuvers on CAM generation moments when $\Delta=200 \cdot \sigma, D=500$

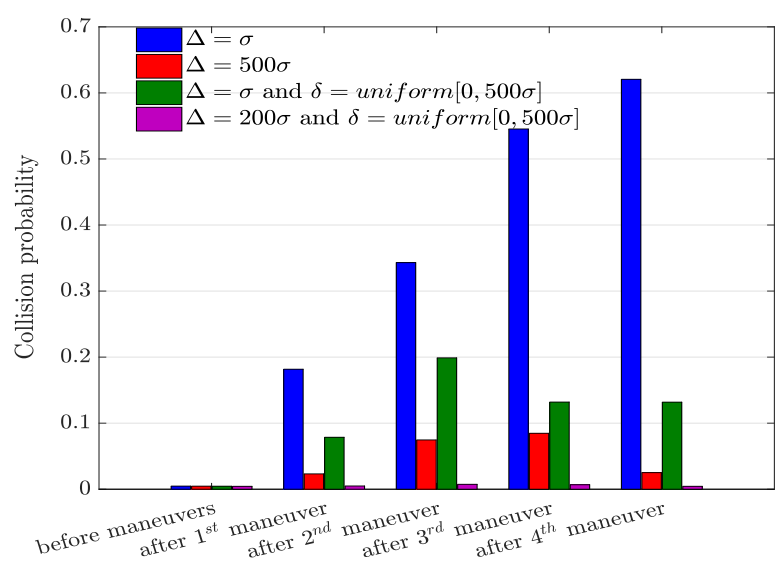

Fig. 18: CAM collision probability

desynchronization component can completely eliminate the CAM synchronization effect.

The first option should be implemented carefully, based on the message age requirements of the CACC/platoon control system [26]. The trade-off between how fast a vehicle can detect a kinematic event and the magnitude of the CAM synchronization effect has to be thoroughly evaluated. At the same time, decreasing the level of synchronicity between platoon members contradicts to the spirit of platooning application requirements, which aims at the best possible coordination of all the maneuvers.

It is important to understand, that the nature of the CAM synchronization phenomenon presented in this manuscript is not dependent on any particular form of the platoon's speed curve as long as its mobility follows a deceleration/acceleration pattern. In other words the studied effect will be observed when the platoon decelerates due to any disturbance in front of it (e.g., slower vehicle, speed limit, etc.), which will obviously occur during its operation on the roads. The CAM synchronization phenomenon itself is a result of triggering rules design - in particular the nature of $v_{\text {min }}$ related condition - that leads to CAM synchronization, when the platoon's speed varies.

Moreover, ETSI EN 302 637-2 CAM is only chosen for representative purposes as an existing available standard of the adaptive beaconing based on the originating vehicle's dynamic 


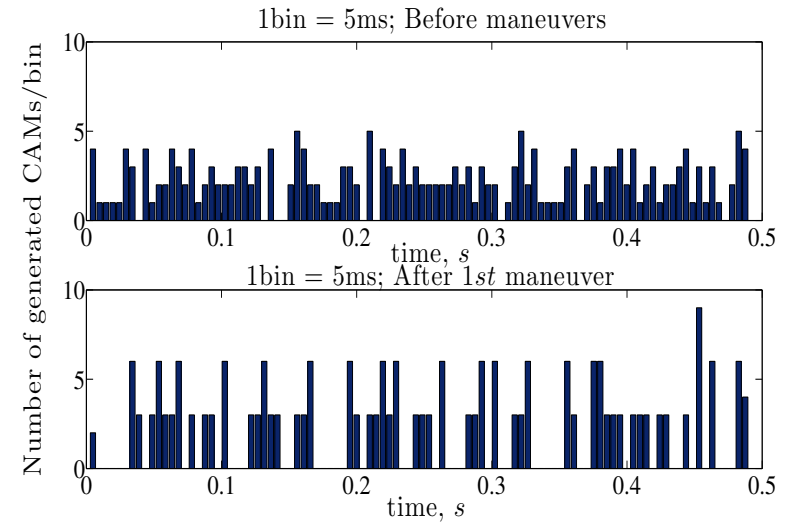

Fig. 19: CAMs grouping after 1 st maneuver

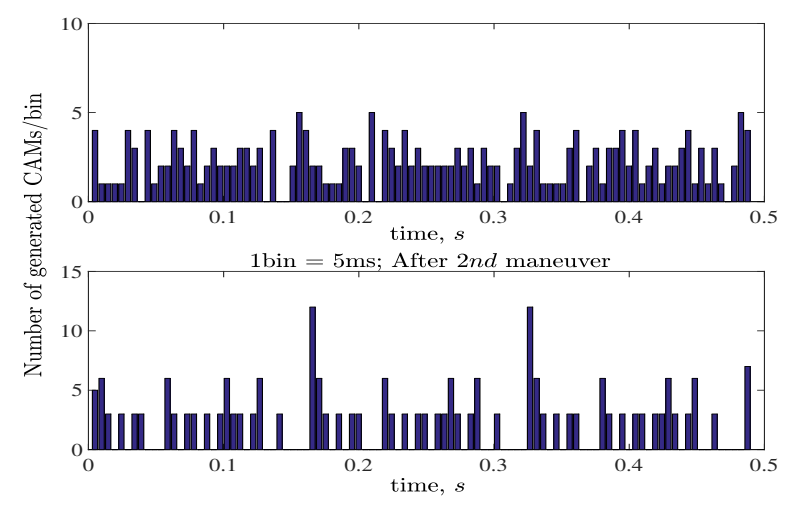

Fig. 20: CAMs grouping after $2 n d$ maneuver

in order to demonstrate the phenomenon of the beacon synchronization effect in a string of vehicles performing cooperative maneuvers. Generally, any adaptive beaconing approach that relies on the track of the speed variation of the originating ITS-S may lead to a similar message synchronization effect in the time domain when vehicles follow mobility scenarios that involve cooperative speed variation.

The discovered negative effect can also influence the functioning of the ETSI congestion control algorithm. This is a subject for our future studies.

\section{ACKNOWLEDGMENT}

This work was partially supported by the Excellence Center at Linkoping-Lund in Information Technology (ELLIIT) strategic research environment (Sweden), NFITS - National ITS Postgraduate School (Sweden) and the "ACDC: Autonomous Cooperative Driving: Communications Issues" project (2014-2016) funded by the Knowledge Foundation (Sweden) in cooperation with Volvo GTT, Volvo Cars, Scania, Kapsch TrafficCom and Qamcom Research \& Technology.

\section{REFERENCES}

[1] "Directive 2010/40/EU of the European parliament and of the council on the framework for the deployment of intelligent transport systems in the field of road transport and for interfaces with other modes of transport," Official Journal of the European Union, 2010.

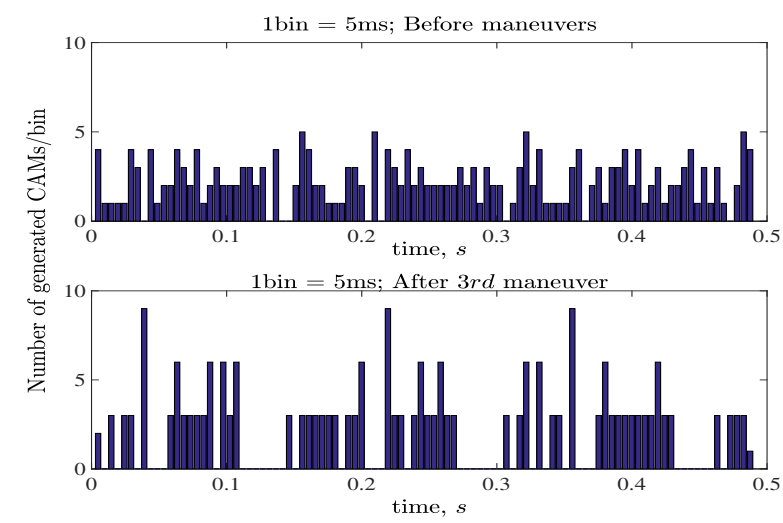

Fig. 21: CAMs grouping after $3 r d$ maneuver

[2] "Intelligent transport systems (ITS); vehicular communications; basic set of applications; part 2: Specification of cooperative awareness basic service," ETSI EN 302 637-2 V1.3.2, 2014.

[3] "Intelligent transport systems (ITS); vehicular communications; basic set of applications; definitions," ETSI TR 102638 V1.1.1, 2009.

[4] N. Lyamin, A. Vinel, and M. Jonsson, "Poster: On the performance of ETSI EN 302 637-2 CAM generation frequency management," in 2014 IEEE Vehicular Networking Conference (VNC), 3-5 December Paderborn, Germany. IEEE Press, 2014, pp. 107-108.

[5] — - "Does ETSI beaconing frequency control provide cooperative awareness?" in 2015 IEEE International Conference on Communications (ICC), IEEE International Conference on, London, UK, June 2015, pp. 10459-10 464.

[6] S. A. A. Shah, E. Ahmed, F. Xia, A. Karim, M. Shiraz, and R. M. Noor, "Adaptive beaconing approaches for vehicular ad hoc networks: A survey," IEEE Systems Journal, vol. pp, no. 99, pp. 1-15, 2016.

[7] C. Sommer, O. K. Tonguz, and F. Dressler, "Traffic information systems: efficient message dissemination via adaptive beaconing," IEEE Communications Magazine, vol. 49, no. 5, pp. 173-179, May 2011.

[8] H. H. Nguyen and H. Y. Jeong, "Crosslayer beaconing design toward guaranteed cooperative awareness with contending traffic," in 2015 IEEE Vehicular Networking Conference (VNC), Dec 2015, pp. 131-134.

[9] M. M. Alotaibi and H. T. Mouftah, "Adaptive expiration time for dynamic beacon scheduling in vehicular ad-hoc networks," in 2015 IEEE 82nd Vehicular Technology Conference (VTC2015-Fall), Sept 2015, pp. $1-6$.

[10] H. P. de Moraes and B. Ducourthial, "Adaptive inter-messages delay in vehicular networks," in 2016 IEEE 12th International Conference on Wireless and Mobile Computing, Networking and Communications (WiMob), Oct 2016, pp. 1-8.

[11] E. Egea-Lopez and P. Pavon-Mario, "Distributed and fair beaconing rate adaptation for congestion control in vehicular networks," IEEE Transactions on Mobile Computing, vol. 15, no. 12, pp. 3028-3041, Dec 2016.

[12] A. Bohm, M. Jonsson, and E. Uhlemann, "Performance comparison of a platooning application using the IEEE 802.11p MAC on the control channel and a centralized MAC on a service channel," in Wireless and Mobile Computing, Networking and Communications (WiMob), 2013 IEEE 9th International Conference on. IEEE, 2013, pp. 545-552.

[13] S. Joerer, M. Segata, B. Bloessl, R. L. Cigno, C. Sommer, and F. Dressler, "A vehicular networking perspective on estimating vehicle collision probability at intersections," IEEE Transactions on Vehicular Technology, vol. 63, no. 4, pp. 1802-1812, May 2014.

[14] M. A. Javed and J. Y. Khan, "A cooperative safety zone approach to enhance the performance of vanet applications," in Vehicular Technology Conference (VTC Spring), 2013 IEEE 77th, June 2013, pp. 1-5.

[15] S. Joerer, M. Segata, B. Bloessl, R. Lo Cigno, C. Sommer, and F. Dressler, "A vehicular networking perspective on estimating vehicle collision probability at intersections," Vehicular Technology, IEEE Transactions on, vol. 63, no. 4, pp. 1802-1812, 2014.

[16] P. M. d'Orey and M. Boban, "Empirical evaluation of cooperative awareness in vehicular communications," in Vehicular Technology Conference (VTC Spring), 2014 IEEE 79th. IEEE, 2014, pp. 1-5.

[17] J. Santa, F. Pereniguez, A. Moragón, and A. F. Skarmeta, "Vehicle- 
to-infrastructure messaging proposal based on CAM/DENM specifications," in Wireless Days (WD), 2013 IFIP. IEEE, 2013, pp. 1-7.

[18] A. Böhm, M. Jonsson, and E. Uhlemann, "Adaptive cooperative awareness messaging for enhanced overtaking assistance on rural roads," in Vehicular Technology Conference (VTC Fall), 2011 IEEE. IEEE, 2011, pp. 1-5.

[19] J. Breu, A. Brakemeier, and M. Menth, "Analysis of cooperative awareness message rates in vanets," in 2013 13th International Conference on ITS Telecommunications (ITST), Nov 2013, pp. 8-13.

[20] T. Lorenzen and H. Tchouankem, "Evaluation of an awareness control algorithm for VANETs based on ETSI EN 302 637-2 v1.3.2," in 2015 IEEE International Conference on Communication Workshop (ICCW), June 2015 , pp. 2458-2464.

[21] C. Bergenhem, "Approaches for facilities layer protocols for platooning," in 2015 IEEE 18th International Conference on Intelligent Transportation Systems. IEEE, 2015, pp. 1989-1994.

[22] M. Boban and P. M. d'Orey, "Exploring the practical limits of cooperative awareness in vehicular communications," IEEE Transactions on Vehicular Technology, vol. 65, no. 6, pp. 3904-3916, June 2016.

[23] "Intelligent transport systems (ITS); decentralized congestion control mechanisms for intelligent transport systems operating in the $5 \mathrm{GHz}$ range; access layer part," ETSI TS 102687 V1.1.1, 2011.

[24] "Intelligent transport systems (ITS); access layer specification for intelligent transport systems operating in the $5 \mathrm{ghz}$ frequency band." ETSI TS 102687 V1.1.1, 2013.

[25] "IEEE std. 802.11-2012, part 11: Wireless LAN medium access control (MAC) and physical layer (PHY) specifications," IEEE Std. 802.112012 , jun 2012.

[26] A. Vinel, L. Lan, and N. Lyamin, "Vehicle-to-vehicle communication in c-acc/platooning scenarios," IEEE Communications Magazine, vol. 53, no. 8, pp. 192-197, 2015.

[27] N. Lyamin, A. V. Vinel, M. Jonsson, and J. Loo, "Real-time detection of denial-of-service attacks in IEEE 802.11p vehicular networks." IEEE Communications letters, vol. 18, no. 1, pp. 110-113, 2014.

[28] M. G. Nilsson, D. Vlastaras, T. Abbas, B. Bergqvist, and F. Tufvesson, "On multilink shadowing effects in measured v2 $\mathrm{v}$ channels on highway," in 2015 9th European Conference on Antennas and Propagation (EuCAP), May 2015, pp. 1-5.

[29] A. Kesting, M. Treiber, and D. Helbing, "Enhanced intelligent driver model to access the impact of driving strategies on traffic capacity," Philosophical Transactions of the Royal Society of London A: Mathematical, Physical and Engineering Sciences, vol. 368, no. 1928, pp. $4585-4605,2010$

[30] M. Segata, S. Joerer, B. Bloessl, C. Sommer, F. Dressler, and R. Lo Cigno, "PLEXE: A Platooning Extension for Veins," in 6th IEEE Vehicular Networking Conference (VNC 2014). Paderborn, Germany: IEEE, December 2014, pp. 53-60.

[31] R. Rajamani, H.-S. Tan, B. K. Law, and W.-B. Zhang, "Demonstration of integrated longitudinal and lateral control for the operation of automated vehicles in platoons," IEEE Transactions on Control Systems Technology, vol. 8, no. 4, pp. 695-708, July 2000.

[32] C. Campolo, A. Molinaro, A. Vinel, and Y. Zhang, "Modeling prioritized broadcasting in multichannel vehicular networks," Vehicular Technology, IEEE Transactions on, vol. 61, no. 2, pp. 687-701, 2012.

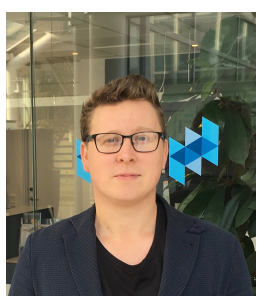

Nikita Lyamin received BS11 (Hons.) and MS13 (Hons.) in telecommunications from Siberian State University of Telecommunications and Information Sciences, Novosibirsk, Russia. He is now a PhD student with the School of Information Technology Halmstad University, Halmstad, Sweden. His current research interests include vehicular ad-hoc networks, ETSI ITS-G5 vehicular communications, $\mathrm{C}-\mathrm{ACC} / \mathrm{Platooning}$ performance evaluation and security in $\mathrm{C}-\mathrm{ACC} / \mathrm{Platooning}$.

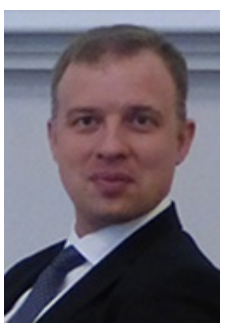

Alexey Vinel (M'07-SM'12) is a Professor with the School of Information Technology, Halmstad University, Sweden (since 2015). He received his $\mathrm{Ph} . \mathrm{D}$. degree in technical sciences from the Institute for Information Transmission Problems, Russia in 2007. His research interests include vehicular networking, cooperative intelligent transportation systems and autonomous driving. He has served as an Associate Editor for IEEE Communications Letters (since 2012) and IEEE Transactions on Vehicular Technology (since 2017).

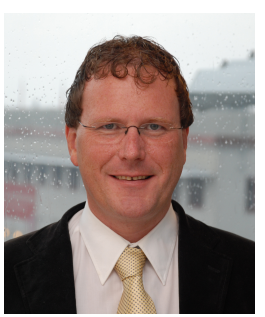

Magnus Jonsson (SM07) received the B.S. and M.S. degrees from Halmstad University, Halmstad, Sweden, in 1993 and 1994, respectively, and the Licentiate of Technology and Ph.D. degrees from Chalmers University of Technology, Gothenburg, Sweden, in 1997 and 1999, respectively, all in computer engineering. Since 2003, he has been a Full Professor of real-time computer systems with Halmstad University, where he is also the Associate Dean with the School of Information Technology (ITE). From 1998 to March 2003, he was an Associate Professor of data communication with Halmstad University (acting between 1998 and 2000). He has published over 130 scientific papers and book chapters, most of them in the areas of vehicular communication, real-time communication, industrial communication, wireless networking, real-time and embedded computer systems, optical networking, and optical interconnection architectures.

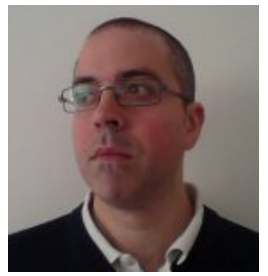

Boris Bellalta is an Associate Professor in the Department of Information and Communication Technologies (DTIC) at Universitat Pompeu Fabra (UPF). He obtained his degree in Telecommunications Engineering from Universitat Politcnica de Catalunya (UPC) in 2002 and the Ph.D. in Information and Communication Technologies from UPF in 2007. His research interests are in the area of wireless networks, with emphasis on the design and performance evaluation of new architectures and protocols. The results from his research have been published in more than 100 international journal and conference papers. He is currently involved in several international and national research projects, including the coordination of the ENTOMATIC FP7 collaborative project. At UPF he is giving several courses on networking, queueing theory and wireless networks. $\mathrm{He}$ is co-designer and coordinator of the interuniversity (UPF and UPC) master degree in Wireless Communications. 